This copy is for your personal, non-commercial use only.

If you wish to distribute this article to others, you can order high-quality copies for your colleagues, clients, or customers by clicking here.

Permission to republish or repurpose articles or portions of articles can be obtained by following the guidelines here.

The following resources related to this article are available online at www.sciencemag.org (this information is current as of January 18, 2013 ):

Updated information and services, including high-resolution figures, can be found in the online version of this article at:

http://www.sciencemag.org/content/338/6112/1314.full.html

Supporting Online Material can be found at:

http://www.sciencemag.org/content/suppl/2012/10/24/science.1229054.DC1.html

This article cites 34 articles, 4 of which can be accessed free:

http://www.sciencemag.org/content/338/6112/1314.full.html\#ref-list-1

This article appears in the following subject collections:

Astronomy

http://www.sciencemag.org/cgi/collection/astronomy 


\section{Binary Millisecond Pulsar Discovery via Gamma-Ray Pulsations}

\author{
H. J. Pletsch, ${ }^{1,2 *}$ L. Guillemot, ${ }^{3}$ H. Fehrmann, ${ }^{1,2}$ B. Allen, ${ }^{1,2,4}$ M. Kramer, ${ }^{3,5}$ C. Aulbert, ${ }^{1,2}$
} M. Ackermann, ${ }^{6}$ M. Ajello, ${ }^{7}$ A. de Angelis, ${ }^{8}$ W. B. Atwood, ${ }^{9}$ L. Baldini, ${ }^{10}$ ]. Ballet, ${ }^{11}$ G. Barbiellini, ${ }^{12,13}$ D. Bastieri, ${ }^{14,15}$ K. Bechtol, ${ }^{7}$ R. Bellazzini, ${ }^{16}$ A. W. Borgland, ${ }^{7}$ E. Bottacini, ${ }^{7}$ T. J. Brandt, ${ }^{17,18}$ ]. Bregeon, ${ }^{16}$ M. Brigida, ${ }^{19,20}$ P. Bruel, $^{21}$ R. Buehler, ${ }^{7}$ S. Buson, ${ }^{14,15}$ G. A. Caliandro ${ }^{22}$ R. A. Cameron, ${ }^{7}$ P. A. Caraveo, ${ }^{23}$ ]. M. Casandjian, ${ }^{11}$ C. Cecchi, ${ }^{24,25}$ Ö. Çelik, ${ }^{26,27,28}$ E. Charles, ${ }^{7}$ R. C. G. Chaves, ${ }^{11}$ C. C. Cheung, ${ }^{29}$ ]. Chiang, ${ }^{7}$ S. Ciprini, ${ }^{25,30}$ R. Claus, ${ }^{7}$ J. Cohen-Tanugi, ${ }^{31}$ ]. Conrad, ${ }^{32,33}$ S. Cutini, ${ }^{34}$ F. D'Ammando, ${ }^{24,35,36}$ C. D. Dermer, ${ }^{37}$ S. W. Digel, ${ }^{7}$ P. S. Drell, ${ }^{7}$ A. Drlica-Wagner, ${ }^{7}$ R. Dubois, ${ }^{7}$ D. Dumora, ${ }^{38}$ C. Favuzzi, ${ }^{19,20}$ E. C. Ferrara, ${ }^{26}$ A. Franckowiak, ${ }^{7}$ Y. Fukazawa, ${ }^{39}$ P. Fusco, ${ }^{19,20}$ F. Gargano, $^{20}$ N. Gehrels, ${ }^{26}$ S. Germani, ${ }^{24,25}$ N. Giglietto, ${ }^{19,20}$ F. Giordano, ${ }^{19,20}$ M. Giroletti, ${ }^{40}$ G. Godfrey, $^{7}$ I. A. Grenier, ${ }^{11}$ M.-H. Grondin, ${ }^{41,42}$ J. E. Grove, ${ }^{37}$ S. Guiriec, ${ }^{26}$ D. Hadasch, ${ }^{22}$ Y. Hanabata, ${ }^{39}$ A. K. Harding, ${ }^{26}$ P. R. den Hartog, ${ }^{7}$ M. Hayashida, ${ }^{7,43}$ E. Hays, ${ }^{26}$ A. B. Hill, ${ }^{7,44}$ X. Hou, ${ }^{45}$ R. E. Hughes, ${ }^{46}$ G. Jóhannesson, ${ }^{47}$ M. S. Jackson, ${ }^{33,48}$ T. Jogler, ${ }^{7}$ A. S. Johnson, ${ }^{7}$ W. N. Johnson, ${ }^{37}{ }^{3}$. Kataoka, ${ }^{49}$ M. Kerr, ${ }^{7}$ J. Knödlseder, ${ }^{17,18}$ M. Kuss, ${ }^{16}{ }^{16}$. Lande, ${ }^{7}$ S. Larsson, ${ }^{32,33,50}$ L. Latronico, ${ }^{51}$ M. Lemoine-Goumard, ${ }^{38}$ F. Longo, ${ }^{12,13}$ F. Loparco, ${ }^{19,20}$ M. N. Lovellette, ${ }^{37}$ P. Lubrano, ${ }^{24,25}$ F. Massaro, ${ }^{7}$ M. Mayer, ${ }^{6}$ M. N. Mazziotta, ${ }^{20}$ J. E. McEnery, ${ }^{26,52}$ ]. Mehault, ${ }^{31}$ P. F. Michelson, ${ }^{7}$ W. Mitthumsiri, ${ }^{7}$ T. Mizuno, ${ }^{53}$ M. E. Monzani, ${ }^{7}$ A. Morselli, ${ }^{54}$ I. V. Moskalenko, ${ }^{7}$ S. Murgia, ${ }^{7}$ T. Nakamori, ${ }^{49}$ R. Nemmen, ${ }^{26}$ E. Nuss, ${ }^{31}$ M. Ohno, ${ }^{55}$ T. Ohsugi, ${ }^{53}$ N. Omodei, ${ }^{7}$ M. Orienti, ${ }^{40}$ E. Orlando, ${ }^{7}$ F. de Palma, ${ }^{19,20}$ D. Paneque, ${ }^{7,56}$ ]. S. Perkins, ${ }^{26,27,28,57}$ F. Piron, ${ }^{31}$ G. Pivato, $^{15}$ T. A. Porter, ${ }^{7}$ S. Rainò, ${ }^{19,20}$ R. Rando, ${ }^{14,15}$ P. S. Ray, ${ }^{37}$ M. Razzano, ${ }^{9,16}$ A. Reimer, ${ }^{7,58}$ O. Reimer, ${ }^{7,58}$ T. Reposeur, ${ }^{38}$ S. Ritz, ${ }^{9}$ R. W. Romani, ${ }^{7}$ C. Romoli, ${ }^{15}$ D. A. Sanchez, ${ }^{41}$ P. M. Saz Parkinson, ${ }^{9}$ A. Schulz, ${ }^{6}$ C. Sgrò, ${ }^{16}$ E. do Couto e Silva, ${ }^{7}$ E. J. Siskind, ${ }^{59}$ D. A. Smith, ${ }^{38}$ G. Spandre, ${ }^{16}$ P. Spinelli, ${ }^{19,20}$ D. J. Suson, ${ }^{60}$ H. Takahashi, ${ }^{39}$ T. Tanaka, ${ }^{7}$ ]. B. Thayer, ${ }^{7}$ J. G. Thayer, ${ }^{7}$ D. J. Thompson, ${ }^{26}$ L. Tibaldo, ${ }^{14,15}$ M. Tinivella, ${ }^{16} \mathrm{E}^{2}$ Troja, $^{26} \mathrm{~T}^{2}$ L. Usher, ${ }^{7}$ J. Vandenbroucke, ${ }^{7}$ V. Vasileiou, ${ }^{31}$ G. Vianello, ${ }^{7,61}$ V. Vitale, ${ }^{54,62}$ A. P. Waite, ${ }^{7}$ B. L. Winer, ${ }^{46}$ K. S. Wood, ${ }^{37}$ M. Wood, ${ }^{7}$ Z. Yang, ${ }^{32,33}$ S. Zimmer $^{32,33}$

Millisecond pulsars, old neutron stars spun up by accreting matter from a companion star, can reach high rotation rates of hundreds of revolutions per second. Until now, all such "recycled" rotation-powered pulsars have been detected by their spin-modulated radio emission. In a computing-intensive blind search of gamma-ray data from the Fermi Large Area Telescope (with partial constraints from optical data), we detected a 2.5-millisecond pulsar, PSR ]1311-3430. This unambiguously explains a formerly unidentified gamma-ray source that had been a decade-long enigma, confirming previous conjectures. The pulsar is in a circular orbit with an orbital period of only 93 minutes, the shortest of any spin-powered pulsar binary ever found.

A lmost exactly 30 years ago, radio observations detected the first neutron star with a millisecond spin period (1). Launched in 2008, the Large Area Telescope (LAT) on the Fermi Gamma-ray Space Telescope (2) confirmed that many radio-detected millisecond pulsars (MSPs) are also bright gamma-ray emitters (3). In each case, gamma-ray ( 0.1 to $100 \mathrm{GeV}$ ) pulsations were revealed by using rotation parameters obtained from radio telescopes (4) to assign rotational phases to LAT-detected photons.

The Fermi LAT also provides sufficient sensitivity to detect pulsars via direct searches for periodicity in the sparse gamma-ray photons. Such blind searches (5) of LAT data for solitary pulsars have so far unveiled 36 younger gamma-ray pulsars (6-9) with rotation rates between 2 and $20 \mathrm{~Hz}$. In the radio band, all but four of these objects remain completely undetected despite deep follow-up radio searches (10). This is a large are in binary systems, where the additionally unknown orbital parameters can increase the computational complexity by orders of magnitude. Thus, blind searches for binary MSPs were hitherto virtually unfeasible.

We have now broken this impasse, detecting a binary MSP, denoted PSR J1311-3430, in a direct blind search of the formerly unidentified gamma-ray source 2FGL J1311.7-3429, one of the brightest listed in the Fermi-LAT Second Source Catalog [2FGL (13)]. This source also had counterparts in several earlier gamma-ray catalogs and was first registered in data from the Energetic Gamma Ray Experiment Telescope [EGRET (14)] on the Compton Gamma Ray Observatory.

In a search for potential optical counterparts of 2FGL J1311.7-3429, Romani (15) identified a quasi-sinusoidally modulated optical flux with a period of $93 \mathrm{~min}$ and conjectured this to be a "black-widow" pulsar binary system (16). In this interpretation, an MSP strongly irradiates what is left of the donor companion star to eventually evaporate it. This plausibly explained the observed brightness variation resulting from strong heating of one side of the companion by the pulsar radiation. Associating this optical variation with the orbital period of the putative binary system constrained the ranges of orbital search parameters and also confined the sky location for the search. Thus, these constraints made a blind binary-MSP search in LAT data feasible; however, the computational challenge involved remained enormous. To test the binary-MSP hypothesis as the possible nature of 2FGL J1311.7-3429, we developed a method to search the LAT data for pulsations over the entire relevant parameter space.

Under the black-widow interpretation, the search is confined toward the sky location of the potential optical counterpart and the orbit is expected to be circular, leaving a five-dimensional search space. The individual dimensions are spin frequency $f$, its rate of change $\dot{f}$, the orbital period $P_{\text {orb }}$, time of ascending node $T_{\text {asc }}$, and $x=a_{\mathrm{p}} \sin \mathrm{r}$, the projection of the pulsar semimajor axis $a_{\mathrm{p}}$ onto the line of sight with orbital inclination angle $\mathrm{r}$. We designed the blind search to maintain sensitivity to very high pulsar spin frequencies, $f<1.4 \mathrm{kHz}$, and values of $\dot{f}$ typical for MSPs, $-5 \times 10^{-13} \mathrm{~Hz} \mathrm{~s}^{-1}<\dot{f}<0$. Although the optical data constrain $P_{\text {orb }}$ and $T_{\text {asc }}$, the uncertainties are by far larger than the precision necessary for a pulsar detection. This required us to search ranges of $P_{\text {orb }}=5626.0 \pm 0.1 \mathrm{~s}$ and $T_{\text {asc }}=$ $56009.131 \pm 0.012$ MJD (modified Julian days) around the nominal values (15), and $0<x<0.1$ 1t-s (light-seconds).

Searching this five-dimensional parameter space fully coherently given a multiple-year data time span is computationally impossible. To solve this problem, we used the hierarchical (three-staged) search strategy that previously enabled the detection of 10 solitary, younger (i.e., non-MSP) 
pulsars in blind searches of LAT data $(8,9)$, exploiting methods originally developed to detect gravitational waves from pulsars (17-20). Here, we expanded this approach to also search over binary orbital parameters. The first stage of the hierarchical scheme is the most computing-intensive and uses an efficient "semicoherent" method (8), extending the method of Atwood et al. (21). This step involves (incoherently) combining coherent Fourier power computed using a window of $2^{20} \mathrm{~s}$ ( $\sim 12$ days) by sliding the window over the entire LAT data set (hence the term "semicoherent"). In a second stage, significant semicoherent candidates are automatically followed up through a fully coherent analysis made possible because only a small region of parameter space around the candidate is explored. A third stage further refines coherent pulsar candidates by including higher signal harmonics [using the $H$-test $(22,23)]$. The computing cost to coherently follow up a single semicoherent candidate is negligible relative to the total cost of the first stage. Therefore, constructing the search grid of the semicoherent stage as efficiently as possible is of utmost importance.

The key element in constructing an optimally efficient grid for the semicoherent search is a distance metric on the search space $(17-19,24)$. The metric provides an analytic geometric tool measuring the expected fractional loss in signalto-noise ratio $(\mathrm{S} / \mathrm{N})$ squared for any given pulsarsignal location at a nearby grid point. The metric is obtained from a Taylor expansion of the fractional loss to second order around the parameterspace location of a given signal. In contrast to searching for solitary pulsars, a difficulty in the binary case is that the metric components ex-

${ }^{1}$ Max-Planck-Institut für Gravitationsphysik (Albert-EinsteinInstitut), D-30167 Hannover, Germany. ${ }^{2}$ Institut für Gravitationsphysik, Leibniz Universität Hannover, D-30167 Hannover, Germany. ${ }^{3}$ Max-Planck-Institut für Radioastronomie, Auf dem Hügel 69, D-53121 Bonn, Germany. ${ }^{4}$ Department of Physics, University of Wisconsin, Milwaukee, WI 53201, USA. ${ }^{5}$ Jodrell Bank Centre for Astrophysics, School of Physics and Astronomy, University of Manchester, Manchester M13 9PL, UK. ${ }^{6}$ Deutsches Elektronen Synchrotron DESY, D-15738 Zeuthen, Germany. ${ }^{7}$ W. W. Hansen Experimental Physics Laboratory, Kavli Institute for Particle Astrophysics and Cosmology, Department of Physics, and SLAC National Accelerator Laboratory, Stanford University, Stanford, CA 94305, USA. ${ }^{8}$ Dipartimento di Fisica, Università di Udine and Istituto Nazionale di Fisica Nucleare, Sezione di Trieste, Gruppo Collegato di Udine, I-33100 Udine, Italy. ${ }^{9}$ Santa Cruz Institute for Particle Physics, Department of Physics, and Department of Astronomy and Astrophysics, University of California, Santa Cruz, CA 95064, USA. ${ }^{10}$ Università di Pisa and Istituto Nazionale di Fisica Nucleare, Sezione di Pisa, I-56127 Pisa, Italy. ${ }^{11}$ Laboratoire AIM, CEA-IRFU/CNRS/ Université Paris Diderot, Service d'Astrophysique, CEA Saclay, F-91191 Gif sur Yvette, France. ${ }^{12}$ Istituto Nazionale di Fisica Nucleare, Sezione di Trieste, I-34127 Trieste, Italy. ${ }^{13}$ Dipartimento di Fisica, Università di Trieste, I-34127 Trieste, Italy. ${ }^{14}$ Istituto Nazionale di Fisica Nucleare, Sezione di Padova, I-35131 Padova, Italy. ${ }^{15}$ Dipartimento di Fisica e Astronomia "G. Galilei," Università di Padova, I-35131 Padova, Italy. ${ }^{16}$ Istituto Nazionale di Fisica Nucleare, Sezione di Pisa, I-56127 Pisa, Italy. ${ }^{17}$ CNRS, IRAP, F-31028 Toulouse Cedex 4, France. ${ }^{18}$ GAHEC, Université de Toulouse, UPS-OMP, IRAP, Toulouse, France. ${ }^{19}$ Dipartimento di Fisica "M. Merlin" dell'Università e del Politecnico di Bari, I-70126 Bari, Italy. ${ }^{20}$ Istituto Nazionale di Fisica Nucleare, Sezione di Bari, I-70126 Bari, Italy. ${ }^{21}$ Laboratoire Leprince-Ringuet, École Polytechnique, CNRS/IN2P3, plicitly depend on the search parameters (24). Thus, the metric (and so the grid point density required to not miss a signal) changes across orbital parameter space. Constructing a simple lattice with constant spacings would be highly inefficient, resulting in either vast over- or undercovering of large parameter-space regions. We developed a grid construction algorithm (25) that effectively uses the metric formalism. Orbital grid points were first placed at random; then, those that were either too close together or too far apart according to the metric were moved (barycentric shifts), minimizing the maximum possible loss in $\mathrm{S} / \mathrm{N}$ for any pulsar signal across the entire search parameter space. By design, the resulting grid (25) ensured never losing more than $30 \%$ in $\mathrm{S} / \mathrm{N}$ for any signal parameters.

The input LAT data we prepared for this search spanned almost 4 years (1437 days) and include gamma-ray photons with LAT-reconstructed directions within $15^{\circ}$ around the targeted sky position (25). To improve the $\mathrm{S} / \mathrm{N}$ of a putative pulsar signal, we assigned each photon a weight (23) measuring the probability of originating from the conjectured pulsar, computed with a spectral likelihood method (25). The gamma-ray spectrum of 2FGL J1311.7-3429 is best modeled by an exponentially cut-off power law (fig. S1), with spectral parameters reminiscent of other gammaray pulsars (Table 1). The computational work of the search was done on the ATLAS cluster in Hannover, Germany. Soon after initiation, the searching procedure convincingly detected PSR J1311-3430.

Following the blind-search detection, we refined the pulsar parameters further in a timing

Palaiseau, France. ${ }^{22}$ Institut de Ciències de l'Espai (IEEE-CSIC), Campus UAB, 08193 Barcelona, Spain. ${ }^{23}$ INAF-Istituto di Astrofisica Spaziale e Fisica Cosmica, I-20133 Milano, Italy.

${ }^{24}$ Istituto Nazionale di Fisica Nucleare, Sezione di Perugia, I-06123 Perugia, Italy. ${ }^{25}$ Dipartimento di Fisica, Università degli Studi di Perugia, I-06123 Perugia, Italy. ${ }^{26}$ NASA Goddard Space Flight Center, Greenbelt, MD 20771, USA. ${ }^{27}$ Center for Research and Exploration in Space Science and Technology (CRESST) and NASA Goddard Space Flight Center, Greenbelt, MD 20771, USA. ${ }^{28}$ Department of Physics and Center for Space Sciences and Technology, University of Maryland Baltimore County, Baltimore, MD 21250, USA. ${ }^{29}$ National Research Council Research Associate, National Academy of Sciences, Washington, DC 20001, resident at Naval Research Laboratory, Washington, DC 20375, USA. ${ }^{30}$ ASI Science Data Center, I-00044 Frascati (Roma), Italy. ${ }^{31}$ Laboratoire Univers et Particules de Montpellier, Université Montpellier 2, CNRS/IN2P3, Montpellier, France. ${ }^{32}$ Department of Physics, Stockholm University, AlbaNova, SE10691 Stockholm, Sweden. ${ }^{33}$ Oskar Klein Centre for Cosmoparticle Physics, AlbaNova, SE-106 91 Stockholm, Sweden. ${ }^{34}$ Agenzia Spaziale Italiana (ASI) Science Data Center, I-00044 Frascati (Roma), Italy. ${ }^{35}$ IASF Palermo, I-90146 Palermo, Italy. ${ }^{36}$ INAF-Istituto di Astrofisica Spaziale e Fisica Cosmica, I-00133 Roma, Italy. ${ }^{37}$ Space Science Division, Naval Research Laboratory, Washington, DC 20375, USA. ${ }^{38}$ Université Bordeaux 1, CNRS/IN2p3, Centre d'Etudes Nucléaires de Bordeaux Gradignan, F-33175 Gradignan, France. ${ }^{39}$ Department of Physical Sciences, Hiroshima University, Higashi-Hiroshima, Hiroshima 739-8526, Japan. ${ }^{40}$ INAF Istituto di Radioastronomia, I-40129 Bologna, Italy. ${ }^{41}$ Max-Planck-Institut für Kernphysik, D-69029 Heidelberg, Germany. ${ }^{42}$ Landessternwarte, Universität Heidelberg, Königstuhl, D-69117 Heidelberg, Germany. ${ }^{43}$ Department of Astronomy, Graduate School of Science, Kyoto University, Sakyoku, Kyoto 606-8502, Japan. ${ }^{44}$ School of Physics and Astronomy, analysis (26). We obtained pulse times of arrival (TOAs) from subdividing the LAT data into 40 segments of about equal length. We produced a pulse profile for each segment using the initial pulsar parameters, and cross-correlated each pulse profile with a multi-Gaussian template derived from fitting the entire data set to determine the TOAs. We used the Tempo2 software (27) to fit the TOAs to a timing model including sky position, $f, \dot{f}$, and binary-orbit parameters (Fig. 1 and Table 1). We found no statistically significant evidence for orbital eccentricity at the $e<10^{-3}$ level. We measured a marginal evidence for a total proper motion of $8 \pm 3$ milliarcseconds per year. Generally, the observed value of $\dot{f}=-3.198$ $( \pm 0.002) \times 10^{-15} \mathrm{~Hz} \mathrm{~s}^{-1}$ is only an upper limit of the intrinsic frequency change $\dot{f}_{\text {in }}$, because of the Shklovskii effect in which Doppler shifts caused by the proper motion can account for part of $\dot{f}$. Under the assumption that the proper motion of PSR J1311-3430 is small enough to approximate $\dot{f} \approx \dot{f}_{\text {in }}$, we derived further quantities from the pulsar rotational parameters (Table 1).

The rotational ephemeris of PSR J1311-3430 also provided constraints on the companion mass $m_{\mathrm{c}}$ through the binary mass function that combines $x, P_{\text {orb}}$, and the gravitational constant $G$,

$$
\begin{aligned}
f\left(m_{\mathrm{p}}, m_{\mathrm{c}}\right) & =\frac{4 \pi^{2}}{G} \frac{x^{3}}{P_{\mathrm{orb}}^{2}}=\frac{\left(m_{\mathrm{c}} \sin \mathrm{l}\right)^{3}}{\left(m_{\mathrm{c}}+m_{\mathrm{p}}\right)^{2}} \\
& =2.995( \pm 0.003) \times 10^{7} M_{\odot}
\end{aligned}
$$

where $m_{\mathrm{p}}$ is the pulsar mass and $M_{\odot}$ is the mass of the Sun. Typical MSP masses are 1.35 to $2.0 \mathrm{M}$. Assuming $m_{\mathrm{p}}=1.35 M_{\odot}$ and $\mathrm{\imath}=90^{\circ}$ (orbit is

University of Southampton, Highfield, Southampton S017 1B], UK. ${ }^{45}$ Centre d'Études Nucléaires de Bordeaux Gradignan, IN2P3/CNRS, Université Bordeaux 1, BP120, F-33175 Gradignan Cedex, France. ${ }^{46}$ Department of Physics, Center for Cosmology and Astro-Particle Physics, Ohio State University, Columbus, $\mathrm{OH} 43210$, USA. ${ }^{47}$ Science Institute, University of Iceland, IS107 Reykjavik, Iceland. ${ }^{48}$ Department of Physics, Royal Institute of Technology (KTH), AlbaNova, SE-106 91 Stockholm, Sweden. ${ }^{49}$ Research Institute for Science and Engineering, Waseda University, 3-4-1, Okubo, Shinjuku, Tokyo 169-8555, Japan. ${ }^{50}$ Department of Astronomy, Stockholm University, SE-106 91 Stockholm, Sweden. ${ }^{51}$ Istituto Nazionale di Fisica Nucleare, Sezione di Torino, I-10125 Torino, Italy. ${ }^{52}$ Department of Physics and Department of Astronomy, University of Maryland, College Park, MD 20742, USA. ${ }^{53}$ Hiroshima Astrophysical Science Center, Hiroshima University, Higashi-Hiroshima, Hiroshima 739-8526, Japan. ${ }^{54}$ Istituto Nazionale di Fisica Nucleare, Sezione di Roma "Tor Vergata," I-00133 Roma, Italy. ${ }^{55}$ Institute of Space and Astronautical Science, JAXA 3-1-1 Yoshinodai, Chuo-ku, Sagamihara, Kanagawa 252-5210, Japan. ${ }^{56}$ Max-Planck-Institut für Physik, D-80805 München Germany. ${ }^{57}$ Harvard-Smithsonian Center for Astrophysics, Cambridge, MA 02138, USA. ${ }^{58}$ Institut für Astro- und Teilchenphysik and Institut für Theoretische Physik, Leopold-Franzens-Universität Innsbruck, A-6020 Innsbruck, Austria. ${ }^{59}$ NYCB Real-Time Computing Inc., Lattingtown, NY 11560, USA. ${ }^{60}$ Department of Chemistry and Physics, Purdue University Calumet, Hammond, IN 46323, USA. ${ }^{61}$ Consorzio Interuniversitario per la Fisica Spaziale (CIFS), I-10133 Torino, Italy. ${ }^{62}$ Dipartimento di Fisica, Università di Roma "Tor Vergata," I-00133 Roma, Italy.

*To whom correspondence should be addressed. E-mail: holger.pletsch@aei.mpg.de 
edge-on) yields the minimum companion mass, $m_{\mathrm{c}}>8.2 \times 10^{-3} M_{\odot}$, which is only about eight times the mass of Jupiter. By means of Kepler's third law and typical MSP masses $\left(m_{\mathrm{p}} \gg m_{\mathrm{c}}\right)$, the binary separation $a=a_{\mathrm{p}}+a_{\mathrm{c}}$ is accurately approximated by $a=0.75 R_{\odot}\left(m_{\mathrm{p}} / 1.35 M_{\odot}\right)^{1 / 3}$, where $R_{\odot}$ is the radius of the Sun. Thus, PSR J1311-3430 is likely the most compact pulsar binary known.

The compact orbit and the optical flaring events (15) suggest that the pulsar heating is driving a strong, possibly variable, stellar wind of ablated material of the companion. Interactions with the companion wind could affect the gammaray flux observed. In a dedicated analysis (25), we found no evidence for a modulation at the orbital period of the gamma-ray flux or its spectrum.

We also examined the gamma-ray spectral parameters of PSR J1311-3430 as a function of rotational phase (25). Dividing the data into 10 segments according to different rotational-phase intervals, we spectrally analyzed each segment separately. In line with the background estimation in the pulse profile (Fig. 1), we detected significant gamma-ray emission at all phases. The gamma-ray spectrum in the off-pulse phase interval (fig. S2) is better modeled by an exponentially cut-off power law, potentially indicative of magnetospheric origin from the pulsar, rather than by a simple power law, which would more likely suggest intrabinary wind shock emission.

Repeated, sensitive radio searches of the previously unidentified gamma-ray source, including Green Bank Telescope observations at $820 \mathrm{MHz}$, gave no pulsar detection (28). However, material ablated from the companion by the pulsar irradiation might obscure radio pulses. At higher radio frequencies, decreased scattering and absorption resulting in shorter eclipses are observed for other black-widow pulsars (12).

The optical observations provide evidence for strong heating of the pulsar companion that is near filling its Roche lobe (15). With $m_{\mathrm{p}}=$ $1.35 M_{\odot}$ and $\imath=90^{\circ}$, the Roche lobe radius of the companion is, to good approximation (29), $R_{\mathrm{L}}=$ $0.063 R_{\odot}$. The minimum mean density of the Roche lobe-filling companion directly follows from the orbital period (30), $\bar{\rho}=45 \mathrm{~g} \mathrm{~cm}^{-3}$. This is twice the density of the planetary-mass companion of PSR J1719-1438 (31). One scenario for the formation of that system posits an ultracompact $\mathrm{x}$-ray binary with a $\mathrm{He}$ or $\mathrm{C}$ degenerate donor transferring mass to the neutron star. However, van Haaften et al. (32) argue that angular momentum losses through gravitational-wave emission are insufficient to reach the low masses and short period of the PSR J1719-1438 system within the age of the universe. Instead, strong heating to bloat the companion or extra angular momentum loss from a companion evaporative wind are required. An alternative scenario (33) proposes that a combination of angular momentum loss and wind evaporation from an initial companion mass of $2 M_{\odot}$ in a 0.8 -day orbit can bring the system to low masses and short orbital
Table 1. Measured and derived parameters for PSR ]1311-3430, with formal $1 \sigma$ uncertainties (dd, days; hh, hours; mm, minutes; ss, seconds). Spectral parameters are averages over pulse phase.

\section{Parameter}

Right ascension (]2000.0) (hh:mm:ss)

Declination (j2000.0) (dd:mm:ss)

Spin frequency, $f(\mathrm{~Hz})$

Frequency derivative, $\dot{f}\left(10^{-15} \mathrm{~Hz} \mathrm{~s}^{-1}\right)$

Reference time scale

Reference time (MJD)

Orbital period $P_{\text {orb }}$ (d)

Projected pulsar semimajor axis $x$ (lt-s)

Time of ascending node $T_{\text {asc }}$ (MJD)

Eccentricity $e$

Data span (MJD)

Weighted root mean square residual ( $\mu s)$

\section{Derived Quantities}

Companion mass $m_{\mathrm{c}}\left(M_{\odot}\right)$

Spin-down luminosity $\dot{E}\left(\mathrm{erg} \mathrm{s}^{-1}\right)$

Characteristic age $\tau_{\mathrm{c}}$ (years)

Surface magnetic field $B_{\mathrm{S}}(\mathrm{G})$

\section{Gamma-Ray Spectral Parameters}

Photon index, $\Gamma$

Cutoff energy, $E_{\mathrm{c}}(\mathrm{GeV})$

Photon flux above $0.1 \mathrm{GeV}, F\left(10^{-8}\right.$ photons $\left.\mathrm{cm}^{-2} \mathrm{~s}^{-1}\right)$

Energy flux above $0.1 \mathrm{GeV}, G\left(10^{-11} \mathrm{erg} \mathrm{cm}^{-2} \mathrm{~s}^{-1}\right)$
Value

$13: 11: 45.7242 \pm 0.0002$

$-34: 30: 30.350 \pm 0.004$

$390.56839326407 \pm 0.00000000004$

$-3.198 \pm 0.002$

Barycentric Dynamical Time (TDB)

55266.90789575858

$0.0651157335 \pm 0.0000000007$

$0.010581 \pm 0.000004$

$56009.129454 \pm 0.000007$

$<0.001$

54682 to 56119

17
$>0.0082$

$1.9 \times 10^{9}$

$2.3 \times 10^{8}$

$1.8 \pm 0.1$

$3.2 \pm 0.4$

$9.2 \pm 0.5$

$6.2 \pm 0.2$

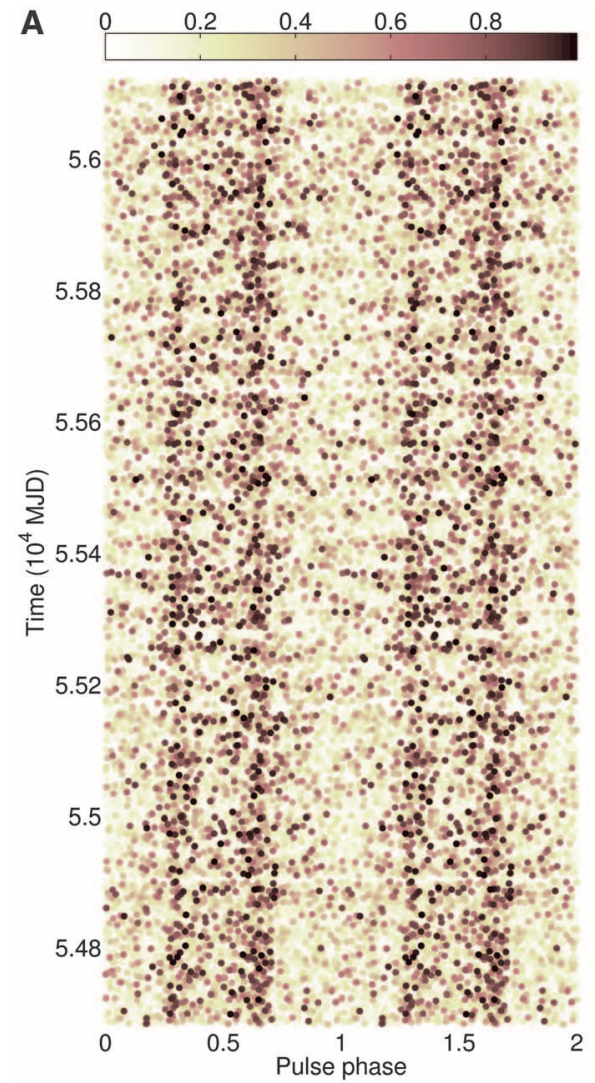

$4.9 \times 10^{34}$
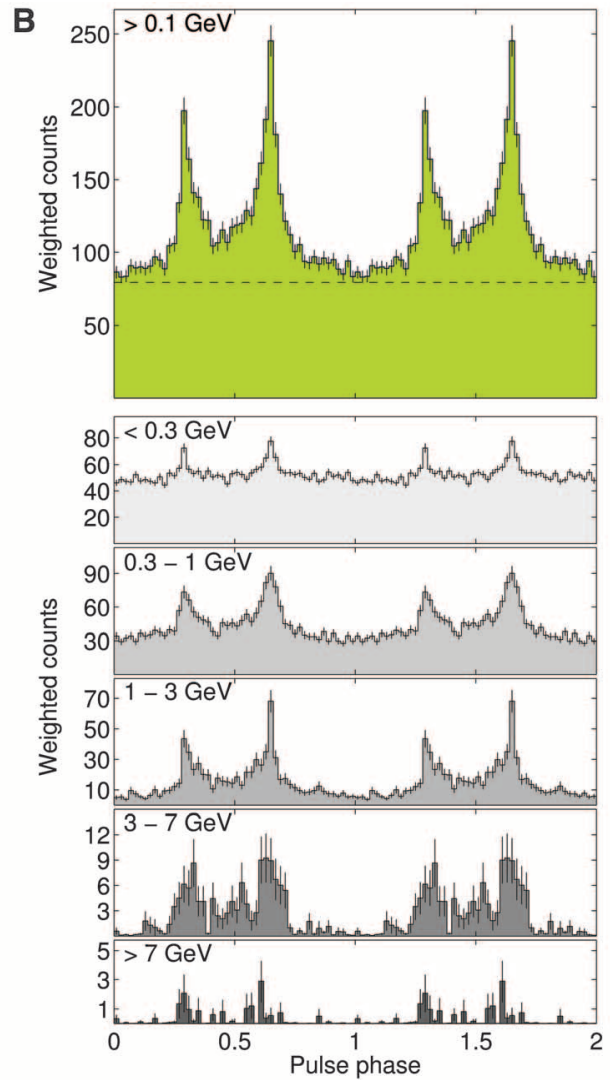

Fig. 1. Phase-time diagram and gamma-ray pulse profiles for PSR J1311-3430. Two pulsar rotations are shown for clarity. (A) The pulsar rotational phase for each gamma-ray photon arrival time; probability weights are shown in color code. (B) The pulse profiles in different energy bands. Each bin is 0.02 in phase, and photon weights are used. The dashed line indicates the estimated background level from a surrounding annular region. 
periods in $\sim 6$ billion years. Indeed, their scenario produces a good match to the $m_{\mathrm{c}} \sim 0.01 M_{\odot}$, $P_{\text {orb }} \sim 0.065$ days seen for PSR J1311-3430. At this point in the evolution the system is detached, the companion is He-dominated, and irradiation has taken over the evolution. Presumably, continued irradiation can drive the system toward PSR J1719-1438-type companion masses, or produce an isolated MSP.

The direct detection of an MSP in a blind search of gamma-ray data implies that further MSPs, including other extreme binary pulsars, may exist among the bright, as yet unidentified 2FGL gamma-ray sources [e.g., $(34,35)]$, which are too radio-faint or obscured by dense companion winds to be found in typical radio searches.

\section{References and Notes}

1. D. C. Backer, S. R. Kulkarni, C. Heiles, M. M. Davis, W. M. Goss, Nature 300, 615 (1982)

2. W. B. Atwood et al., Astrophys. J. 697, 1071 (2009).

3. A. A. Abdo et al., Science 325, 848 (2009).

4. D. A. Smith et al., Astron. Astrophys. 492, 923 (2008).

5. The term "blind search" is used to reflect the fact that the pulsar parameters are unknown a priori, implying that a wide range of pulsar parameters must be explicitly searched.

6. A. A. Abdo et al., Science 325,840 (2009).
7. P. M. Saz Parkinson et al., Astrophys. J. 725, 571 (2010).

8. H. J. Pletsch et al., Astrophys. J. 744, 105 (2012).

9. H. J. Pletsch et al., Astrophys. J. 755, L20 (2012).

10. P. S. Ray et al., http://arxiv.org/abs/1205.3089 (2012).

11. K. P. Watters, R. W. Romani, Astrophys. J. 727, 123 (2011).

12. J. W. T. Hessels et al., Science 311, 1901 (2006).

13. P. L. Nolan et al., Astrophys. J. 199 (suppl.), 31 (2012).

14. C. E. Fichtel et al., Astrophys. J. 94 (suppl.), 551 (1994).

15. R. W. Romani, Astrophys. J. 754, L25 (2012).

16. A. S. Fruchter, D. R. Stinebring, J. H. Taylor, Nature 333, 237 (1988)

17. P. R. Brady, T. Creighton, Phys. Rev. D 61, 082001 (2000).

18. H. J. Pletsch, B. Allen, Phys. Rev. Lett. 103, 181102 (2009).

19. H. J. Pletsch, Phys. Rev. D 82, 042002 (2010).

20. H. J. Pletsch, Phys. Rev. D 83, 122003 (2011).

21. W. B. Atwood, M. Ziegler, R. P. Johnson, B. M. Baughman, Astrophys. J. 652, L49 (2006).

22. O. C. de Jager, B. C. Raubenheimer, J. W. H. Swanepoel, Astron. Astrophys. 221, 180 (1989).

23. M. Kerr, Astrophys. J. 732, 38 (2011).

24. C. Messenger, Phys. Rev. D 84, 083003 (2011).

25. See supplementary materials on Science Online.

26. P. S. Ray et al., Astrophys. J. 194 (suppl.), 17 (2011).

27. G. B. Hobbs, R. T. Edwards, R. N. Manchester, Mon. Not. R. Astron. Soc. 369, 655 (2006).

28. S. M. Ransom et al., Astrophys. J. 727, L16 (2011).

29. B. Paczyński, Annu. Rev. Astron. Astrophys. 9, 183 (1971).

30. J. Frank, A. R. King, D. J. Raines, Accretion Power in Astrophysics (Cambridge Univ. Press, Cambridge, 1985).
31. M. Bailes et al., Science 333, 1717 (2011).

32. L. M. van Haaften, G. Nelemans, R. Voss, P. G. Jonker, Astron. Astrophys. 541, A22 (2012).

33. O. G. Benvenuto, M. A. De Vito, ]. E. Horvath, Astrophys. J. 753, L33 (2012)

34. R. W. Romani, M. S. Shaw, Astrophys. J. 743, L26 (2011).

35. M. Ackermann et al., Astrophys. J. 753, 83 (2012).

Acknowledgments: This work was supported by the Max-Planck-Gesellschaft. The Fermi LAT Collaboration acknowledges support from several agencies and institutes for both development and the operation of the LAT as well as scientific data analysis. These include NASA and Department of Energy (United States), CEA/Irfu and IN2P3/CNRS (France), ASI and INFN (Italy), MEXT, KEK, and JAXA (Japan), and the K. A. Wallenberg Foundation, the Swedish Research Council, and the National Space Board (Sweden). Additional support from INAF in Italy and CNES in France for science analysis during the operations phase is also gratefully acknowledged. Fermi LAT data are available from the Fermi Science Support Center (http://fermi.gsfc.nasa.gov/ssc)

\section{Supplementary Materials}

www.sciencemag.org/cgi/content/full/science.1229054/DC1 Materials and Methods

Figs. $\mathrm{S} 1$ and $\mathrm{S} 2$

References (36-38)

20 August 2012; accepted 17 October 2012

Published online 25 October 2012;

$10.1126 /$ science. 1229054

\section{Mapping Local Charge Recombination Heterogeneity by Multidimensional Nanospectroscopic Imaging}

Wei Bao, ${ }^{1,2 *}$ M. Melli, ${ }^{1 *}$ N. Caselli, ${ }^{3,4}$ F. Riboli, ${ }^{3,4}$ D. S. Wiersma, ${ }^{3,5}$ M. Staffaroni, ${ }^{6}$ H. Choo, ${ }^{7}$ D. F. Ogletree, ${ }^{1}$ S. Aloni, ${ }^{1}$ ]. Bokor, ${ }^{1,6}$ S. Cabrini, ${ }^{1} \dagger$ F. Intonti, ${ }^{3,4}$ M. B. Salmeron, ${ }^{1,2}$ E. Yablonovitch, ${ }^{6}$ P. J. Schuck, ${ }^{1} \dagger$ A. Weber-Bargioni ${ }^{1} \dagger$

As materials functionality becomes more dependent on local physical and electronic properties, the importance of optically probing matter with true nanoscale spatial resolution has increased. In this work, we mapped the influence of local trap states within individual nanowires on carrier recombination with deeply subwavelength resolution. This is achieved using multidimensional nanospectroscopic imaging based on a nano-optical device. Placed at the end of a scan probe, the device delivers optimal near-field properties, including highly efficient far-field to near-field coupling, ultralarge field enhancement, nearly background-free imaging, independence from sample requirements, and broadband operation. We performed $\sim 40$-nanometer-resolution hyperspectral imaging of indium phosphide nanowires via excitation and collection through the probes, revealing optoelectronic structure along individual nanowires that is not accessible with other methods.

$\mathrm{I}$ n the study of materials, optical microscopy maps the spatial distribution of a specific quantity (such as morphology), whereas optical spectroscopy provides physical and chemical material properties (such as electronic structure). An ongoing challenge to understanding matter at the nanoscale is the difficulty in carrying out local optical spectroscopy. On a fundamental level, this should be possible by squeezing light beyond the diffraction limit (1-4). Optical antennabased geometries have been designed to address this nanospectroscopy imaging problem by trans- forming light from the far field to the near field, but with limitations on sensitivity, bandwidth, resolution, and/or sample types (5).

We report a strategy that overcomes these limitations, based on a geometry that is capable of efficiently coupling far-field light to the near field and vice versa, without background illumination over a wide range of wavelengths. The geometry consists of a three-dimensional (3D) tapered structure terminating in a nanometer-sized gap (Fig. 1A), with a shape resembling that of a "campanile" bell tower (hereafter referred to as campanile). We demonstrated with the campanile probe hyperspectral imaging of local optoelectonic properties in indium phosphide nanowires (InP NWs) by exciting and collecting signal through the probe tip. InP NWs, with their direct solar spectrum-matched bandgap and presumed low surface recombination velocity, are expected to be the central functional elements of nextgeneration light-harvesting devices. With the campanile probe, we collect full spectra at each pixel in a scan image, revealing photoluminescence (PL) heterogeneity along individual NWs by mapping local charge recombination originating from trap states: critical optoelectronic information that was unobtainable with previous methods.

Various near-field probe geometries have been engineered with extraordinary optical transmission (6) or with coupled optical antenna structures $(7,8)$ directly on the scanning-probe apex, greatly improving coupling efficiencies as compared to conventional aperture-based probes $(9)$

${ }^{1}$ Molecular Foundry, Lawrence Berkeley National Laboratory, Berkeley, CA 94720, USA. ²Department of Materials Science and Engineering, University of California Berkeley, Berkeley, CA 94720, USA. ${ }^{3}$ European Laboratory for Non-Linear Spectroscopy, 50019 Sesto Fiorentino, Firenze, Italy. ${ }^{4}$ Dipartimento di Fisica e Astronomia, Università di Firenze, 50019 Sesto Fiorentino, Firenze, Italy. ${ }^{5}$ Istituto Nazionale di Ottica (CNRINO), 50125 Firenze, Italy. ${ }^{6}$ Department of Electrical Engineering and Computer Sciences, University of California Berkeley, Berkeley, CA 94720-1770, USA. ${ }^{7}$ Department of Electrical Engineering, California Institute of Technology, Pasadena, CA 91125, USA.

*These authors contributed equally to this work.

†To whom correspondence should be addressed. E-mail: afweber-bargioni@lbl.gov (A.W.-B.); scabrini@lbl.gov (S.C.); pjschuck@lbl.gov (P.J.S.) 